\title{
Die aktuelle Behandlung der Obstipation im Kindesalter
}

\author{
Olivia Liem $^{\mathrm{a}, \mathrm{b}}$ Carlo Di Lorenzo $^{\mathrm{b}} \quad$ Jan A.J.M. Taminiau ${ }^{\mathrm{a}}$ Hayat M. Mousa ${ }^{\mathrm{b}}$ \\ Marc A. Benninga ${ }^{a}$ \\ a Department of Pediatric Gastroenterology and Nutrition, Emma Children's Hospital, Academic Medical Center, \\ Amsterdam, The Netherlands, and ' Division of Pediatric Gastroenterology, Columbus Children's Hospital, \\ Columbus, Ohio, USA
}

\section{Schlüsselwörter \\ Obstipation $\cdot$ Behandlung $\cdot$ Kinder}

\section{Zusammenfassung}

Bei der Obstipation im Kindesalter handelt es sich um ein globales Problem. Die Obstipation ist eine der weiter verbreiteten Erkrankungen, mit der sowohl Kinderärzte im Allgemeinen als auch pädiatrische Gastroenterologen konfrontiert sind. Die Behandlung der chronischen Obstipation stellt eine Herausforderung dar und erfordert häufig eine langfristige Nachsorge und Medikamenteneinnahme. Trotz der hohen Prävalenz der Erkrankung wurden bisher nur wenige randomisierte Studien durchgeführt, um die Wirksamkeit der diversen Interventionen zur Behandlung dieser Erkrankung zu untersuchen. Dieser Übersichtsartikel erläutert, welche Optionen es derzeit zur Behandlung der Obstipation im Kindesalter gibt und geht dabei auf einige der am häufigsten gestellten Fragen und gängigsten falschen Vorstellungen von Ärzten und Eltern ein.

Copyright $\odot 2008$ Nestec Ltd., Vevey/S. Karger AG, Basel

\section{Einleitung}

Die Obstipation ist ein weit verbreitetes Problem und betrifft Millionen von Kindern weltweit. Eine vor Kurzem durchgeführte systematische Literaturanalyse zeigt, dass die Prävalenz der Obstipation in Industrienationen und in Entwicklungsländern zwischen 7 und 29,6\% beträgt [1]. Bei ungefähr 95\% der Kinder mit Obstipation lassen sich keine offensichtlichen anatomischen, biochemischen oder physiologischen Ursachen feststellen [2]. Viele dieser Kinder leiden unter funktioneller Obstipation, die wahrscheinlich die Folge einer Stuhlretention nach schmerzhafter bzw. unangenehmer Stuhlentleerung ist.

Die meisten Kinder benötigen eine Langzeitbehandlung und ungefähr 30\% der Kinder leiden auch nach der Pubertät noch unter den Symptomen der Obstipation, wie z.B. seltener und schmerzhafter Stuhlentleerung und Enkopresis [3]. Es überrascht daher nicht, dass sich diese chronischen Darmbeschwerden signifikant und dauerhaft auf das Selbstwertgefühl und die emotionale Reifung und Entwicklung des Kindes auswirken können. Die Obstipation muss daher zur Vermeidung der mit ihr einhergehenden Morbidität frühzeitig und wirksam behandelt werden [4].

Da bei Kindern zu wenige randomisierte kontrollierte Studien durchgeführt wurden, beruht die Behandlung der Obstipation grösstenteils auf klinischen Erfahrungen und nicht auf evidenzbasierten Studien. Eine akut auftretende einfache Obstipation wird auf herkömmliche Weise mit ballaststoffreicher Ernährung bei ausreichender Flüssigkeitszufuhr, dem Führen eines Stuhltagebuchs und Toilettentraining behandelt. Die vor Kurzem aktualisierten Empfehlungen der nordamerikanischen Gesellschaft für pädiatrische Gastroenterologie (NASPGHAN) sprechen bei der Behandlung der chronischen Obstipati-

\section{KARGER \\ Fax +4161306 1234 \\ E-Mail karger@karger.ch}

www.karger.com
C 2008 Nestec Ltd, Vevey/S. Karger AG, Basel

0250-9652/07/0652-0073\$23.50/0

Online-Zugriff auf:

www.karger.com/and
O. Liem, MD

Columbus Children's Hospital

Department of Pediatric Gastroenterology and Nutrition

700 Children's Drive, Columbus, OH 43205 (USA)

Tel. +1 614722 3421, Fax +1 614722 3454, E-Mail liemo@chi.osu.edu 
on von vier wichtigen Phasen: (1) Aufklärung; (2) Desimpaktion; (3) Prävention einer erneuten Stuhlimpaktion und (4) Nachsorge [5]. Dieser Übersichtsartikel erläutert diese vier Phasen und geht dabei auf einige der im $\mathrm{Zu}$ sammenhang mit der Obstipation im Kindesalter am häufigsten gestellten Fragen und gängigsten falschen Vorstellungen ein.

\section{Aufklärung und Beratung}

Die Aufklärung und Unterstützung von Eltern und Kindern ist eine der Hauptsäulen der Behandlung der funktionellen Obstipation. Die Eltern müssen beschwichtigt und über die Bandbreite der normalen Stuhlganghäufigkeit, die Ätiologie und die Prävalenz der Obstipation im Kindesalter beraten werden. Bei Stuhlinkontinenz ist es wichtig, den Erziehungsberechtigten klar zu machen, dass dieses lästige Symptom durch Überlaufenkopresis und nicht durch absichtlich aufsässiges Verhalten verursacht wird. Die Eltern sollten den betroffenen Kindern keine Schuldzuweisungen machen, sondern sie positiv bestärken und motivieren. Vor Behandlungsbeginn sollte den Eltern und Kindern versichert werden, dass eine vollständige Heilung bei angemessener, häufig längerfristiger Behandlung möglich ist. Man sollte betonen, dass der Zeitpunkt des Behandlungserfolgs häufig nicht vorhergesagt werden kann, dass es bei $50 \%$ der behandelten Patienten innerhalb des ersten Jahres zu einem Rückfall kommt und dass die Dauer der Erhaltungstherapie normalerweise zwischen 6 und 24 Monate beträgt [3].

Da Abführmittel (Laxantien) für gewöhnlich eine wichtige Rolle bei der Behandlung spielen, ist die Aufklärung der Eltern über die verabreichten Medikamente von wesentlicher Bedeutung, denn falsche Vorstellungen über Laxantien, die sich negativ auf die Compliance auswirken könnten, sind weit verbreitet. Während die zur Behandlung von anderen Erkrankungen, wie z.B. von gastroösophagealem Reflux, Asthma, Bluthochdruck und sonstigen chronischen Krankheiten, eingesetzten Medikamente von den Familien der betroffenen Kinder in der Regel akzeptiert und manchmal sogar gefordert werden, stehen die Erziehungsberechtigten der Verordnung von Laxantien für gewöhnlich ablehnend gegenüber, da sie die Vorstellung, dass ein so kleines Kind bereits Abführmittel benötigen könnte, schrecklich finden! Eine der am häufigsten von besorgten Eltern gestellten Fragen lautet, ob eine Langzeitbehandlung mit Abführmitteln schädlich ist und zu 'Darmträgheit' und körper- licher Abhängigkeit führen kann. Eine vor Kurzem durchgeführte Bewertung der bestehenden Literatur hat sich detailliert mit diesen Bedenken auseinandergesetzt [6]. Die Argumente, Abführmittel (insbesondere stimulierende Laxantien) würden das autonome Nervensystem des Dickdarms schädigen, beruhten auf Daten aus mangelhaft dokumentierten Versuchen, während die professionell und mit Hilfe einer Reihe verschiedener Methoden durchgeführten Studien keine Hinweise auf eine solche Schädigung ergaben [6]. Osmotische Laxantien scheinen keine langfristigen Nebenwirkungen zu haben. Manche Patienten mit chronischer Obstipation müssen zwar, um eine normale Stuhlentleerung zu erzielen, langfristig mit Laxantien behandelt werden, aber es gibt keinerlei Hinweise darauf, dass dieser Umstand auf eine frühere Einnahme von Abführmitteln zurückzuführen ist oder dass es nach Einstellen der Behandlung mit Abführmitteln zu Rebound-Symptomen kommt. Man sollte den Eltern daher versichern, dass die Medikamente bei ordnungsgemässer Einnahme gut verträglich sind und sie ermutigen, die verordnete Behandlung zu befolgen, damit die Therapie erfolgreich ist.

\section{Ernährung}

Trotz der weit verbreiteten Auffassung, die Obstipation werde häufig durch Ballaststoffmangel verursacht, wurden bisher nur wenige pädiatrische Studien durchgeführt, welche die Wirksamkeit einer Therapie mit Ballaststoffen bewerteten. Über die Ballaststoffaufnahme von Kindern mit Obstipation gibt es widersprüchliche Aussagen. Während manche Berichte von einer niedrigeren Ballaststoffaufnahme als bei Kindern ohne Obstipation sprechen, melden andere eine vergleichbare oder sogar höhere Ballaststoffaufnahme [7-9]. Die empfohlene Ballaststoffaufnahme beträgt bei Kindern ab 2 Jahren das Lebensalter in Jahren plus $5 \mathrm{~g}$ [10].

Standardisierte Studien jüngeren Datums, die an Kindern durchgeführt wurden, haben bei Kindern mit chronischer Verstopfung eine Nutzwirkung der Supplementierung von Ballaststoffen belegt. Castillejo et al. [11] fanden heraus, dass sich bei Kindern mit grundlegend verlängerter Kolontransitzeit, die eine Supplementierung von Ballaststoffen in Form von Kakaoschalen erhielten, die Kolontransitzeit im Vergleich zu einer Placebogruppe verkürzte. Sie beobachteten ferner, dass der Prozentsatz an Patienten, die über eine harte Stuhlkonsistenz klagten, abnahm. Hier ist anzumerken, dass die durchschnittliche Ballaststoffaufnahme dieser unter Obstipation leidenden 
Kinder in etwa der für diese Altersgruppe empfohlenen Menge entsprach. Eine Supplementierung von Glucomannan führte ebenfalls zur häufigeren Entleerung weicherer Stühle und sorgte ferner dafür, dass die Patienten besser auf eine Behandlung mit Laxantien ansprachen $[12,13]$. Die schlechte Compliance mit der Behandlung, die darauf zurückzuführen ist, dass die meisten Ballaststoffe, wie z.B. Zellulosefasern, Guargummi und Pektinfasern, in grossen Mengen konsumiert werden müssen, um eine Wirkung zu entfalten und nicht sehr schmackhaft sind, ist einer der Hauptgründe dafür, dass die langfristige Wirksamkeit von Ballaststoffen bei Kindern begrenzt ist. Die in den oben erwähnten Studien eingesetzten Faserstoffe wurden gut akzeptiert und hatten keine nennenswerten Nebenwirkungen.

Während ein Mangel an Ballaststoffen im Allgemeinen wahrscheinlich nicht ursächlich für die chronische Obstipation ist, kann er bei einer Untergruppe von Patienten durchaus einer der Faktoren sein, die zur Obstipation beitragen. Aus diesem Grund wird bei Obstipation zur Unterstützung der Behandlung eine ausgewogene Ernährung mit Vollkornprodukten, Obst und Gemüse empfohlen und nicht zwanghaft eine ballaststoffreiche Ernährung eingeführt.

Eine erhöhte Flüssigkeitszufuhr wird zur Behandlung der Obstipation ebenfalls häufig empfohlen. Diese Empfehlung beruht auf der Annahme, eine erhöhte Flüssigkeitszufuhr führe zu mehr Flüssigkeit im Darm und dies wiederum würde die Stuhlentleerung fördern. Eine Studie, in der 108 Kinder mit Obstipation in eine Kontrollund zwei Behandlungsgruppen randomisiert wurden, von denen eine Gruppe angewiesen wurde, ihre tägliche Wasseraufnahme um 50\% zu erhöhen, ergab keine Änderungen hinsichtlich Stuhlhäufigkeit, Stuhlkonsistenz oder problemloser Stuhlentleerung [14]. Die Ursache dafür, dass eine erhöhte Flüssigkeitszufuhr nicht zu einer Veränderung der Stuhlgewohnheiten führte, ist möglicherweise darin zu suchen, dass sich das Resorptionsvermögen des Dünn- und Dickdarms bei akuten und chronischen Herausforderungen als extrem anpassungsfähig erweist $[15,16]$. Ausserdem sind es die gelösten Substanzen und nicht das Wasser, welche die Ausscheidungen aus dem Ileum beeinflussen. Geringfügige Änderungen der Flüssigkeitsaufnahme wirken sich daher nicht signifikant auf die Stuhlkonsistenz aus. Belege dafür, dass sich die Obstipation durch eine erhöhte Flüssigkeitsaufnahme erfolgreich behandeln lässt, gibt es nicht. Liegen keine Anzeichen einer Dehydratation vor, sollten Kinder daher nicht gezwungen werden, mehr als gewöhnlich zu trinken.

Die aktuelle Behandlung der Obstipation im Kindesalter

\section{Desimpaktion}

Vor Beginn der Erhaltungstherapie wird eine Desimpaktion der für gewöhnlich vorhandenen Stuhlmassen empfohlen, um nach Beginn der Behandlung verstärkte Bauchschmerzen und Stuhlinkontinenz aufgrund von Überlaufenkopresis zu vermeiden [5]. In unkontrollierten klinischen Studien wurde belegt, dass eine erfolgreiche Desimpaktion oral oder rektal bzw. durch eine Kombination dieser beiden Methoden möglich ist [1720]. Eine Gegenüberstellung dieser Methoden der Desimpaktion in randomisierten kontrollierten Studien erfolgte bislang nicht. Eine mit verschiedenen Dosierungen durchgeführte randomisierte Doppelblindstudie ergab, dass Polyethylenglykol (PEG) in einer Dosierung von 1$1,5 \mathrm{~g} / \mathrm{kg} /$ Tag bei Kindern zur Desimpaktion wirksam ist und von Kindern und Eltern akzeptiert wird [19-21]. Die Untersuchungen der Serumosmolalität, Elektrolyte oder Nierenfunktion ergaben keine Anomalien. Weitere Agenzien, die erfolgreich eingesetzt, bisher aber noch nicht in kontrollierten Studien untersucht wurden, sind u.a. Magnesiumhydroxid, Magnesiumcitrat, Laktulose, Sorbitol, Senna und Bisacodyl.

Die rektale Desimpaktion erfolgt häufig mit Hilfe von phosphat- oder salzhaltigen Klistieren oder einem Paraffinöl-Klistier mit anschliessendem Phosphatklysma. Sowohl die orale als auch die rektale Desimpaktion haben gewisse Vor- und Nachteile. Die orale Methode ist zwar weniger invasiv, nimmt dafür aber mehr Zeit in Anspruch als die rektale Methode. Bei phosphathaltigen Klistieren sollte die Ausscheidung überwacht werden, um eine übermässige Phosphatresorption zu vermeiden [22]. Die zur Wahl stehenden Optionen sollten am besten zuerst gemeinsam besprochen und die Behandlung dann zusammen mit der Familie und dem Kind gewählt werden.

\section{Erhaltungstherapie}

Ziel nach der Desimpaktion ist es, ein- bis zweimal täglich für eine schmerzlose Entleerung von weichem Stuhl zu sorgen. Die regelmässige Stuhlentleerung über einen längeren Zeitraum ist wichtig, um eine erneute Impaktion und Stuhlretention zu vermeiden. Dies erfordert für gewöhnlich eine medikamentöse Behandlung mit Laxantien in Kombination mit einer Verhaltenstherapie über einen längeren Zeitraum, manchmal über Monate oder sogar Jahre. Trotz der hohen Prävalenz und der Chronizität der Obstipation wurden nur wenige rando-

Ann Nestlé [Ger] 2007;65:73-80 
misierte kontrollierte Studien durchgeführt, um die Wirksamkeit einer medikamentösen Behandlung mit Laxantien bei Kindern zu bewerten [23-25].

$\mathrm{Zu}$ den osmotischen Laxantien zählen Magnesiummilch, Lactulose und PEG. Der Wirkmechanismus der osmotischen Laxantien beruht entweder auf Salzen mit im Darm schlecht resorbierbaren Kat- und Anionen (Magnesium, Phosphat), Molekülen, die im Dickdarm nicht resorbiert, jedoch zu einem gewissen Grad abgebaut werden (Lactulose), oder Verbindungen, die nicht metabolisiert werden. Das osmotische Laxans Magnesiumhydroxid/-sulfat, das auch unter der Bezeichnung 'Magnesiummilch' bekannt ist, blickt auf eine lange Geschichte zurück. Magnesiumhydroxid und Magnesiumsulfat sind zwei Salze, die im Darm schlecht resorbiert werden und deren Wirkmechanismus entweder auf Osmose beruht oder auf einer sekretagogen Wirkung auf die Prostaglandine oder das Cholecystokinin, durch welche die Motilität des Dickdarms verbessert wird [26, 27]. Die Lactulose, ein weiteres häufig eingesetztes $\mathrm{Ab}$ führmittel, ist ein aus der Lactose gewonnenes Disaccharid, das wirksam die Häufigkeit der Stuhlentleerung erhöht und die Stuhlbeschaffenheit normalisiert [28, 29].

Polyethylenglykole (PEG) sind synthetische, nicht resorbierbare Verbindungen mit hoher molekularer Masse (3000 und mehr), die von den Bakterien des Dickdarms nicht abgebaut werden. Sie entfalten ihren Wirkmechanismus über Osmose und Volumenausdehnung im Dickdarm. PEG ist als Pulver erhältlich und ist farb- und geruchlos. Angeboten werden reines Polyethylenglykol oder Polyethylenglykol in Kombination mit Elektrolyten. Reines PEG ist geschmacksneutral, was sich in der Langzeitbehandlung von Kindern zur Steigerung der Compliance als signifikanter Vorteil erweisen kann. Zwei kontrollierte randomisierte Multicenter-Studien bescheinigten einer Behandlung mit PEG in Kombination mit Elektrolyten eine höhere kurzfristige Erfolgsrate als einer Behandlung mit Lactulose (50 vs. 29\%). Die mit PEG behandelten Patienten wiesen ferner eine bessere Stuhlbeschaffenheit auf, hatten mehr Appetit, litten seltener unter Stuhlimpaktion und benötigten auch seltener Klistiere. Bei einer Behandlung mit PEG in Kombination mit Elektrolyten traten signifikant weniger unerwünschte Arzneimittelwirkungen, wie Bauchschmerzen, Übelkeit, Blähungen und Durchfall, auf als bei einer Behandlung mit Lactulose, aber die Kinder beklagten sich über den schlechten Geschmack. Eine an 49 Kindern mit funktioneller Obstipation und Stuhlinkontinenz durchgeführte randomisierte Studie stellte eine
Behandlung mit reinem PEG (ohne Elektrolyte) einer Therapie mit Magnesiummilch gegenüber und ergab bei den nach 1, 3, 6 und 12 Monaten durchgeführten Followup-Untersuchungen eine ähnliche Wirksamkeit: häufigere Stuhlentleerung, Rückgang der Stuhlinkontinenz und Abnahme der Bauchschmerzen [25]. Die Studie zeigte ferner, dass reines PEG besser schmeckte und besser verträglich war als Magnesiummilch (die Einnahme der Magnesiummilch wurde von 33\% der Kinder verweigert, die PEG-Einnahme dagegen von keinem Kind). Unerwünschte Arzneimittelwirkungen während der PEG-Behandlung wurden nicht gemeldet. Die Eltern sollten darauf vorbereitet werden, dass die Stuhlinkontinenz nach Einleitung einer Behandlung mit Laxantien anfänglich anhalten oder sich sogar verschlimmern kann, insbesondere wenn das Kind Angst vor der Stuhlentleerung hat oder sich der Stuhlentleerung weiterhin verweigert.

Stimulierende Laxantien, wie Senna und Bisacodyl, wurden schon vor Jahrzehnten entwickelt, so dass die zur Untersuchung dieser Arzneimittel durchgeführten Studien nicht den aktuellen Kriterien für qualitativ hochwertige klinische Studien entsprechen, was eine Bewertung für eine Vergleichsanalyse unmöglich macht [30]. Im Gegensatz zu den zuvor erwähnten osmotischen Laxantien wird die tägliche Einnahme dieser stimulierenden Laxantien über einen längeren Zeitraum im Allgemeinen nicht empfohlen. $\mathrm{Zu}$ den häufig auftretenden unerwünschten Arzneimittelwirkungen zählen u.a. Krämpfe. Die Einnahme dieser Medikamente kann zeitweilig notwendig werden, um ein erneutes Auftreten der Impaktion zu vermeiden. Nach wie vor umstritten ist, ob Laxantien, die Anthraquinon enthalten, wie z.B. Senna, morphologische Veränderungen des autonomen Nervensystems des Dickdarms verursachen können. Bei regelmässiger Einnahme kann es innerhalb von Monaten zu Melanosis coli, einer gut sichtbaren braunen Verfärbung des Dickdarms, kommen, die auch Monate nach Absetzen des Abführmittels weiterhin erkennbar ist [31, 32]. Verursacht wird diese Pigmentierung dadurch, dass die Makrophagen in der Submukosa laxantienverfärbte Zelldebris aufnehmen. Die Verfärbung scheint sich jedoch nicht auf die Funktion auszuwirken [33]. Ein vernünftiger Therapieansatz besteht darin, täglich osmotische Laxantien zu verabreichen und diese bei Bedarf, d.h. wenn nach über 48 oder 72 Stunden keine Stuhlentleerung stattgefunden hat, mit stimulierenden Laxantien zu kombinieren. 


\section{Verhaltenstherapie}

Eine Therapie bestehend aus Verhaltensintervention in Kombination mit einer Behandlung mit Laxantien zielt darauf ab, die seelische Belastung zu reduzieren, die für das Kind mit der Darmentleerung verbunden ist und über positive Bestärkung normale Stuhlgewohnheiten $\mathrm{zu}$ entwickeln bzw. wiederherzustellen. In der Praxis geht man häufig wie folgt vor: Das Kind wird zu einem regelmässigen Toilettentraining ermutigt und angewiesen, nach jeder Mahlzeit (um den Magen-Dickdarm-Reflex zu nutzen) 5 bis 10 Minuten lang auf der Toilette zu sitzen und zu versuchen, Stuhl abzusetzen. Der Patient wird angespornt, seine Füsse auf eine Fussstütze zu stellen und aktiv zu pressen. Das Kind muss verstehen, dass der Schlüssel zum Erfolg darin liegt, dem Stuhldrang Folge zu leisten und nicht darin, den Stuhl zurückzuhalten. Ein weiteres, ebenfalls häufig eingesetztes Hilfsmittel ist das Führen eines Stuhltagebuchs für das betroffene Kind, um Beschwerden aufzuzeichnen, den Behandlungsfortschritt zu quantifizieren und die Motivation zu verbessern. Zur Sicherstellung einer guten Compliance werden kleine, altersentsprechende Belohnungen eingeführt.

Die Verhaltensinterventionen zur Änderung des Verhaltens wurden an Kindern mit retentiver Enkopresis untersucht. Eine an 87 Kindern durchgeführte, randomisierte kontrollierte Studie verglich die folgenden drei Behandlungsmodalitäten: intensive medikamentöse Behandlung, intensive medikamentöse Behandlung plus verbessertes Toilettentraining und intensive medikamentöse Behandlung plus verbessertes Toilettentraining und Biofeedbacktherapie. Nach zwölfmonatiger Behandlung wurden bezüglich der Erfolgsrate keine statistisch signifikanten Unterschiede zwischen den drei Methoden festgestellt [34]. Eine Studie zur Untersuchung, wie sich eine zusätzlich zur medikamentösen Behandlung mit Laxantien durchgeführte Verhaltenstherapie auswirkt, wurde bisher nicht durchgeführt.

Bei Kindern, bei denen eine intensive medizinische Behandlung nicht anschlägt, bzw. bei Kindern mit schweren emotionalen oder ernsten familiären Problemen ist eine Überweisung zum Psychologen indiziert.

\section{Biofeedback}

Die Methode des Biofeedbacks scheint bei der Behandlung der Obstipation im Kindesalter nur eine begrenzte Rolle zu spielen [35]. Mit dieser auf Bestärkung basierenden Methode der Konditionierung, die sich aus einer psychologischen Lerntheorie ableitet, wird Kindern vermittelt, wie sie ihre Sphinktermuskeln mit Hilfe von Biofeedbacksystemen kontrollieren können, z.B. Geräten zur Überwachung der anorektalen Kontinenz, mit deren Hilfe sich der Muskeltonus des äusseren Schliessmuskels auf einem Bildschirm oder in Form von Klangmodulationen darstellen lässt [36]. Das Grundprinzip dieses Verfahrens beruht auf der Tatsache, dass sich der äussere Schliessmuskel und die Beckenbodenmuskulatur bei über $50 \%$ der Kinder mit Defäkationsstörungen während der Stuhlentleerung nicht entspannen, sondern zusammenziehen (physiologische Manifestation der Stuhlretention) [37]. Biofeedback gilt auch als nützlich, um Kindern ein Gefühl für die rektale Distension zu vermitteln, da einigen Kindern nicht bewusst ist, dass dieses Gefühl den Stuhldrang signalisiert.

In kleineren unkontrollierten Studien wurde belegt, dass sich mit Hilfe des Biofeedbacktrainings eine angemessene Kontraktion des äusseren Schliessmuskels bzw. eine Normalisierung der abnormen Defäkationsmuster erzielen lässt. Während eine von Loening-Baucke durchgeführte Studie [38, 39] anfänglich auf eine signifikante kurzfristige Wirkung des Biofeedback-Verfahrens hindeutete, wurde in anderen kontrollierten Studien und nach einer langfristigen Nachsorgebehandlung kein zusätzlicher Nutzen zu einer Behandlung mit Laxantien nachgewiesen [37, 40, 41]. Laut einer grossen randomisierten kontrollierten Studie, die an 192 Kindern mit Obstipation durchgeführt wurde und belegte, dass das Erzielen eines normalen Defäkationsmusters nicht mit klinischem Erfolg gleichzusetzen war, scheint sich die beobachtete Verbesserung der manometrischen Parameter ferner nicht in einer funktionellen Verbesserung auszudrücken. Aufgrund dieser Daten kamen wir zu dem Schluss, dass es keine überzeugenden Belege gibt, die dafür sprechen, dass die Biofeedback-Methode bei der Mehrzahl der Kinder eine nützliche Zusatztherapie zur Behandlung von Defäkationsstörungen darstellt.

\section{Chirurgische Eingriffe}

Zwar führen die oben beschriebenen konventionellen Behandlungsprotokolle bei den meisten Kindern mit Obstipation zum Erfolg, aber bei einigen Kindern persistieren die Symptome trotz intensiver Therapie und optimaler Compliance. Sind die Symptome so ausgeprägt, 
dass sie die Lebensqualität des Patienten signifikant beeinträchtigen, sollten die Ärzte möglicherweise einen chirurgischen Eingriff vornehmen. Insbesondere die häufig mit der chronischen Obstipation einhergehende Enkopresis kann sich äusserst negativ auf die emotionale und soziale Entwicklung der betroffenen Kinder auswirken [42]. Bis vor Kurzem beschränkten sich die chirurgischen Optionen bei Fehlschlagen der konservativen Behandlung auf die Anlage eines Kolostomas bzw. die Durchführung einer subtotalen Kolektomie. Diese chirurgischen Interventionen galten als zu invasiv, waren häufig irreversibel, wurden oft mit unberechenbaren klinischen Outcomes assoziiert und kamen daher selten zum Einsatz.

Inzwischen gibt es weitere Alternativen. Ein kontinentes Appendikostoma oder ein Button-Coecostoma können angelegt werden, um eine antegrade Darmspülung zu ermöglichen, die den gesamten Darm säubern und zuverlässig für Intervalle ohne Enkopresis sorgen soll. Die Röhrchen des Coecostomas können mittels interventioneller Radiologie oder Endoskopie anstatt durch Operation sicher perkutan platziert werden [43]. Regelmässige Dickdarm-Spülungen steigern die Defäkationshäufigkeit signifikant, führen zu einer Abnahme der Enkopresis und erhöhen somit die Lebensqualität [43, 44]. In den meisten Studien werden hohe Erfolgsraten (von bis zu 80\%) erzielt, wahrscheinlich weil die Patienten vor Durchführung der Coecostomie sorgfältig bewertet und ausgewählt werden. Man geht davon aus, dass die Methode bei älteren motivierten Kindern, die normal auf die auf den Dickdarm wirkenden Stimulanzien ansprechen, und bei Kindern, bei denen die Manometrie des Dickdarms eine höhere Zahl an Kontraktionen zeigt, erfolgreicher ist als bei jüngeren Kindern, die nicht auf die auf den Dickdarm wirkenden Stimulanzien ansprechen [45]. Die Manometrie des Dickdarms wird bei Kindern mit Defäkationsstörungen zur Vorhersage der Erfolgschancen einer Coecostomie eingesetzt. Zu den möglichen $\mathrm{Ne}$ benwirkungen einer Appendikostomie bei Kindern zählen u.a. die Stomastenose (11\%), eine schmerzvolle oder schwierige Katheterisierung (5\%) und die iatrogene Perforierung des Appendikostomas [46]. Zu den bekannten Komplikationen, die im Zusammenhang mit der Stomaversorgung auftreten, zählen verrutschte Röhrchen (26\%), Leckagen am Stoma (42\%) und Granulationsgewebe (68\%) [43].

Selbstverständlich sollte eine Operation nur bei Kindern erwogen werden, die seit vielen Jahren unter diesen Symptomen leiden, auf diverse medikamentöse Behandlungen nicht angesprochen haben und zur Behandlung ihrer Symptome bereits mehrmals ins Krankenhaus eingewiesen werden mussten. Eine umfassende Untersuchung der Motilität, u.a. durch Manometrie des Dickdarms, erweist sich bei der klinischen Entscheidungsfindung bezüglich der Operation möglicherweise als hilfreich [47-49].

\section{Nachsorge und Überweisung an Spezialisten}

Die Erhaltungsphase beginnt, nachdem die ersten beiden Schritte, die Aufklärung und Desimpaktion, abgeschlossen sind und kann monate- oder jahrelang dauern. Van Ginkel et al. [3] fanden heraus, dass es nach dem anfänglichen Behandlungserfolg bei mindestens 50\% der behandelten Kinder innerhalb der ersten 5 Jahre zu mindestens einem Rückfall kam, dass die Krankheit bei 3050\% der Patienten persistierte und dass diese Patienten auch nach fünfjähriger Nachsorgebehandlung, manchmal sogar in einem Alter von über 18 Jahren, unter schweren Symptomen litten. Diese hohe Rückfallrate und die Persistenz der Obstipation unterstreichen, wie wichtig eine sorgfältige und langfristige Nachsorge bei unter Obstipation leidenden Kindern ist. Es wird empfohlen, betroffene Kinder während der Nachsorgebehandlung anfangs 3 bis 4 Mal wöchentlich in der Praxis zu untersuchen, um die Einhaltung und die Wirksamkeit des Behandlungsprogramms sicherzustellen. Stellt sich ein Behandlungserfolg ein, können die Intervalle der Verlaufskontrolle verlängert und die Praxisbesuche im Wechsel mit telefonischer Beratung durchgeführt werden. Die Aufklärung und Entmystifizierung der Erkrankung muss während der Behandlung möglicherweise mehrmals wiederholt werden [50].

Nach erfolgreicher Behandlung stellt sich die Frage, ob die medikamentöse Behandlung fortgesetzt werden soll, und wenn ja, für wie lange. Angesichts der hohen Rückfallraten in den ersten Jahren wird die Behandlung in vielen Fällen, nachdem der Patient symptomfrei ist, mindestens 2 bis 3 Monate lang fortgesetzt und erst dann ein langsames Ausschleichen aus der Behandlung versucht. Bleibt das Kind auch weiterhin symptomfrei, kann die Dosis gesenkt werden, wobei die Eltern angewiesen werden müssen, die Darmgewohnheiten und die Beschaffenheit des Stuhls ihres Kindes genauestens zu überwachen, um schmerzhafte Stuhlentleerungen zu vermeiden, die zu einer erneuten Stuhlimpaktion führen könnten. Bei erneutem Auftreten der Symptome sollten nicht nur die Medikamente wieder eingenommen, sondern auch wieder ein Stuhltagebuch geführt 
und erneut Wert auf das Toilettentraining gelegt werden.

Schlägt die Therapie fehl bzw. bestehen Bedenken bezüglich einer organischen Erkrankung, dann sollte das Kind an einen pädiatrischen Gastroenterologen überwiesen bzw. ein solcher Spezialist zu Rate gezogen werden. Der pädiatrische Gastroenterologe kann das Kind erneut untersuchen, spezielle Tests anordnen, falls er dies für notwendig erachtet, und die früheren Behandlungen überprüfen. Häufig werden andere oder weitere Medikamente bzw. eine höhere Dosis der bereits eingenommenen Medikamente verordnet, bevor zusätzliche Untersuchungen durchgeführt werden.

\section{Hauptaussagen}

(1) Die Aufklärung der Kinder und ihrer Eltern über normale Darmgewohnheiten, die Ätiologie der Obstipation und ihre Behandlung ist ein wichtiger Faktor, um die Einhaltung des Behandlungsprogramms zu gewährleisten und ein erfolgreiches Outcome zu erzielen.

(2)Es sprechen überzeugende Belege dafür, PEG bei Obstipation im Kindesalter als Arzneimittel der Wahl einzusetzen.

(3) Angesichts der hohen Rückfallraten und der Persistenz der Symptome ist eine sorgfältige und langfristige Nachsorge bei Kindern mit Obstipation von grosser Bedeutung.

\section{Literatur}

$\checkmark 1$ van den Berg MM, Benninga MA, Di Lorenzo C: Epidemiology of childhood constipation: a systematic review. Am J Gastroenterol 2006;101:2401-2409.

-2 Baker SS, Liptak GS, Colletti RB, et al: Constipation in infants and children: evaluation and treatment. A medical position statement of the North American Society for Pediatric Gastroenterology and Nutrition. J Pediatr Gastroenterol Nutr 1999;29:612-626.

-3 van Ginkel R, Reitsma JB, Buller HA, et al: Childhood constipation: longitudinal follow-up beyond puberty. Gastroenterology 2003;125:357-363.

4 Loening-Baucke V: Constipation in early childhood: patient characteristics, treatment, and longterm follow up. Gut 1993;34: 1400-1404.

5 Evaluation and treatment of constipation in infants and children: recommendations of the North American Society for Pediatric Gastroenterology, Hepatology and Nutrition. J Pediatr Gastroenterol Nutr 2006;43: e1-e13.

6 6 Muller-Lissner SA, Kamm MA, Scarpignato C, Wald A: Myths and misconceptions about chronic constipation. Am J Gastroenterol 2005; 100:232-242.

$\checkmark 7$ Roma E, Adamidis D, Nikolara R, et al: Diet and chronic constipation in children: the role of fiber. J Pediatr Gastroenterol Nutr 1999;28:169-174.

8 McClung HJ, Boyne L, Heitlinger L: Constipation and dietary fiber intake in children. Pediatrics 1995;96:999-1000.

$\checkmark 9$ Morais MB, Vitolo MR, Aguirre AN, Fagundes-Neto U: Measurement of low dietary fiber intake as a risk factor for chronic constipation in children. J Pediatr Gastroenterol Nutr 1999;29:132-135.
10 Williams CL, Bollella M, Wynder EL: A new recommendation for dietary fiber in childhood. Pediatrics 1995;96:985-988.

11 Castillejo G, Bullo M, Anguera A, et al: A controlled, randomized, double-blind trial to evaluate the effect of a supplement of cocoa husk that is rich in dietary fiber on colonic transit in constipated pediatric patients. Pediatrics 2006;118:e641-e648.

-12 Loening-Baucke V, Miele E, Staiano A: Fiber (glucomannan) is beneficial in the treatment of childhood constipation. Pediatrics 2004; 113:e259-e264.

13 Staiano A, Simeone D, Del GE, et al: Effect of the dietary fiber glucomannan on chronic constipation in neurologically impaired children. J Pediatr 2000;136:41-45.

$\checkmark 14$ Young RJ, Beerman LE, Vanderhoof JA: Increasing oral fluids in chronic constipation in children. Gastroenterol Nurs 1998;21: 156-161.

15 Schlessinger M, Fordtran JS (eds): Gastroenterology, ed 6. Philadelphia, Saunders, 1998, pp 1451-1471.

16 Debongnie JC, Phillips SF: Capacity of the human colon to absorb fluid. Gastroenterology 1978;74:698-703.

17 Tolia V: Use of a balanced lavage solution in the treatment of fecal impaction. J Pediatr Gastroenterol Nutr 1988;7:299-301.

18 Tolia V, Lin CH, Elitsur Y: A prospective randomized study with mineral oil and oral lavage solution for treatment of faecal impaction in children. Aliment Pharmacol Ther 1993;7:523-529.

19 Youssef NN, Peters JM, Henderson W, et al: Dose response of PEG 3350 for the treatment of childhood fecal impaction. J Pediatr 2002; 141:410-414.
20 Pashankar DS, Uc A, Bishop WP: Polyethylene glycol 3350 without electrolytes: a new safe, effective, and palatable bowel preparation for colonoscopy in children. J Pediatr 2004;144:358-362.

21 Candy DC, Edwards D, Geraint M: Treatment of faecal impaction with polyethelene glycol plus electrolytes $(\mathrm{PGE}+\mathrm{E})$ followed by a double-blind comparison of PEG + E versus lactulose as maintenance therapy. J Pediatr Gastroenterol Nutr 2006;43:65-70.

22 McClung HJ, Potter C: Rational use of laxatives in children. Adv Pediatr 2004;51:231262.

23 Voskuijl W, de Lorijn F, Verwijs W, et al: PEG 3350 (Transipeg) versus lactulose in the treatment of childhood functional constipation: a double blind, randomised, controlled, multicentre trial. Gut 2004;53:1590-1594.

24 Dupont C, Leluyer B, Ammar F, et al: Double-blind randomized evaluation of clinical and biological tolerance of polyethylene glycol 4000 versus lactulose in constipated children. J Pediatr Gastroenterol Nutr 2005;41:625-633.

25 Loening-Baucke V, Pashankar DS: A randomized, prospective, comparison study of polyethylene glycol 3350 without electrolytes and milk of magnesia for children with constipation and fecal incontinence. Pediatrics 2006;118:528-535.

26 Donowitz M, Rood RP: Magnesium hydroxide: new insights into the mechanism of its laxative effect and the potential involvement of prostaglandin E2. J Clin Gastroenterol 1992;14:20-26.

27 Younoszai MK, Tolaymat N: Chronic functional constipation in infants and children; in Lebenthal E (ed): Textbook of Gastroenterology and Nutrition in Infancy. New York, Raven Press Ltd, 1989, pp 1311-1326. 
28 Martino AM, Pesce F, Rosati U: The effects of lactitol in the treatment of intestinal stasis in childhood (in Italian). Minerva Pediatr 1992;44:319-323.

29 Pitzalis G, Deganello F, Mariani P, et al: Lactitol in chronic idiopathic constipation in children. Pediatr Med Chir 1995;17:223226.

30 Price KJ, Elliott TM: What is the role of stimulant laxatives in the management of childhood constipation and soiling? Cochrane Database Syst Rev 2001;3:CD002040.

> 31 Willems M, van Buuren HR, de Krijger R: Anthranoid self-medication causing rapid development of melanosis coli. Neth J Med 2003;61:22-24.

-32 Speare GS: Melanosis coll; experimental observations on its production and elimination in twenty-three cases. Am J Surg 1951;82: 631-637.

33 Ghadially FN, Walley VM: Melanoses of the gastrointestinal tract. Histopathology 1994; 25:197-207.

-34 Borowitz SM, Cox DJ, Sutphen JL, Kovatchev B: Treatment of childhood encopresis: a randomized trial comparing three treatment protocols. J Pediatr Gastroenterol Nutr 2002; 34:378-384.

35 Brazzelli M, Griffiths P: Behavioural and cognitive interventions with or without other treatments for the management of faecal incontinence in children. Cochrane Database Syst Rev 2006;2:CD002240.
36 Engel BT, Nikoomanesh P, Schuster MM: Operant conditioning of rectosphincteric responses in the treatment of fecal incontinence. N Engl J Med 1974;290:646-649.

37 van der Plas RN, Benninga MA, Buller HA, et al: Biofeedback training in treatment of childhood constipation: a randomised controlled study. Lancet 1996;348:776-780.

38 Loening-Baucke V: Modulation of abnormal defecation dynamics by biofeedback treatment in chronically constipated children with encopresis. J Pediatr 1990;116:214222.

39 Loening-Baucke V: Biofeedback treatment for chronic constipation and encopresis in childhood: long-term outcome. Pediatrics 1995;96:105-110.

40 Wald A, Chandra R, Gabel S, Chiponis D: Evaluation of biofeedback in childhood encopresis. J Pediatr Gastroenterol Nutr 1987; 6:554-558.

41 Nolan T, Catto-Smith T, Coffey C, Wells J: Randomised controlled trial of biofeedback training in persistent encopresis with anismus. Arch Dis Child 1998;79:131-135.

42 Youssef NN, Langseder AL, Verga BJ, Mones RL, Rosh JR: Chronic childhood constipation is associated with impaired quality of life: a case-controlled study. J Pediatr Gastroenterol Nutr 2005;41:56-60.

43 Mousa HM, van den Berg MM, Caniano DA, et al: Cecostomy in children with defecation disorders. Dig Dis Sci 2006;51:154-160.
44 Youssef NN, Barksdale JE, Griffiths JM, et al: Management of intractable constipation with antegrade enemas in neurologically intact children. J Pediatr Gastroenterol Nutr 2002;34:402-405.

45 van den Berg MM, Hogan M, Caniano DA, et al: Colonic manometry as predictor of cecostomy success in children with defecation disorders. J Pediatr Surg 2006;41:730-736.

46 Cascio S, Flett ME, De la Hunt M, et al: MACE or caecostomy button for idiopathic constipation in children: a comparison of complications and outcomes. Pediatr Surg Int 2004;20:484-487.

47 Youssef NN, Pensabene L, Barksdale E Jr, Di Lorenzo C: Is there a role for surgery beyond colonic aganglionosis and anorectal malformations in children with intractable constipation? J Pediatr Surg 2004;39:73-77.

48 Villarreal J, Sood M, Zangen T, et al: Colonic diversion for intractable constipation in children: colonic manometry helps guide clinical decisions. J Pediatr Gastroenterol Nutr 2001;33:588-591.

49 Pensabene L, Youssef NN, Griffiths JM, Di Lorenzo C: Colonic manometry in children with defecatory disorders. Role in diagnosis and management. Am J Gastroenterol 2003; 98:1052-1057.

50 Rappaport LA, Levine MD: The prevention of constipation and encopresis: a developmental model and approach. Pediatr Clin North Am 1986;33:859-869. 